\title{
Association Between Cholesterol and 2,3-Diphosphoglycerate in Genetically Selected Hooded Rat Lines
}

\author{
N. A. Noble, ${ }^{1,2}$ G. J. Brewer, ${ }^{1}$ and F. J. Oelshlegel, Jr. ${ }^{1,3}$ \\ Received 13 Sept. 1976-Final 5 July 1977
}

We have developed two strains of hooded rats with differing erythrocyte oxygen affinities by selection on red cell 2,3-diphosphoglycerate levels. Genetic studies have shown that these strains differ at one DPG-level-determining locus. This article reports the results of a study which involved measurement of plasma cholesterol levels in rats from the strains and the $F_{2}$ progeny of strain intercrosses. Low-DPG strain rats, with high oxygen affinity, had significantly higher mean cholesterol levels than High-DPG rats. Animals from the extremes of the $F_{2}$ distribution of DPG levels showed similar, significantly different mean cholesterol levels, indicating that the negative association between DPG and cholesterol levels in strain rats was not due to inadvertent fixation of unrelated genes during selection on DPG. The possibility is discussed that high oxygen affinity, brought about by low DPG levels, may be causative in increasing cholesterol levels.

KEY WORDS: cholesterol ; 2,3-diphosphoglycerate; oxygen affinity; hooded rats.

\section{INTRODUCTION}

We have developed two rat strains by genetic selection on blood levels of the red cell glycolytic intermediate 2,3-diphosphoglycerate (DPG). Beginning

This work was supported by an NIH Training Grant (5-T01-GM-0071) and a Michigan Heart Association Grant.

${ }^{1}$ Department of Human Genetics, University of Michigan Medical School, Ann Arbor, Michigan 48109.

${ }^{2}$ Present address: Department of Medicine, Harbor General Hospital, Torrance, California 90502.

${ }^{3}$ Present address: Department of Preventive Medicine, University of Mississippi, Jackson, Mississippi 39216. 
with an outbred population of about 100 hooded (Long-Evans) rats, two-way selection for high and low levels of DPG resulted in production of a HighDPG rat strain and a Low-DPG rat strain where mean DPG levels were markedly different after three generations of selection (Noble and Brewer, 1972). Intercross of generation 10 High-DPG and Low-DPG strain rats followed by mating of $F_{1}$ animals produced an $F_{2}$ generation where the distribution of DPG levels fit closely to a 3 High-DPG:1 Low-DPG ratio. The conclusion of this work (Noble and Brewer, 1977) was that the DPG differences between the strains are due to two alleles at one locus, where the High-DPG strain allele is partially dominant over the Low-DPG strain allele.

It has been shown that DPG plays a major role in determining red cell oxygen affinity by binding to hemoglobin and lowering oxygen affinity (Benesch and Benesch, 1967; Chanutin and Curnish, 1967). Therefore, an animal with low DPG levels will have higher hemoglobin oxygen affinity than an animal with high DPG levels, assuming that other factors influencing oxygen affinity are not different. The DPG differences between the Low-DPG and High-DPG rat strains resulted in marked differences in oxygen affinity (mean p50 values, a measure of oxygen affinity, were 34 and $41 \mathrm{~mm} \mathrm{Hg}$ for Low-DPG and High-DPG strain animals, respectively, Brewer et al., 1972). Other factors which could influence in vivo oxygen affinity, including whole blood $p \mathrm{H}$, carbon dioxide tension (measured on mixed venous blood), and arterial oxygen tension, did not differ significantly between these strains (unpublished data).

We felt that these rat strains, with large differences in hemoglobin oxygen affinity, would be excellent material to further test the idea that hemoglobin oxygen affinity is an important pathogenic element in arteriosclerosis (Brewer, 1972). In the present article we report interesting differences in plasma cholesterol levels in the rat strains and the $F_{2}$ progeny of the intercross.

\section{METHODS}

Animals in this study were of two general types. The first type was animals of the High-DPG and Low-DPG strains, after at least seven generations of selection on DPG levels so that animals used were almost certainly homozygous for the High-DPG and Low-DPG alleles, respectively. The second type was derived from intercross of the High-DPG strain with the Low-DPG strain. The $F_{1}$ progeny were then mated, and the $F_{2}$ progeny studied. The $\mathrm{F}_{2}$ segregated into High-DPG and Low-DPG types, and their cholesterol levels were evaluated. The genetics of the DPG system in these animals is the topic of another article (Noble and Brewer, 1977).

Nonfasting blood for DPG, hemoglobin, and plasma cholesterol level determinations was drawn into heparinized syringes from anesthetized 
animals by cardiac puncture. Blood for all DPG and hemoglobin determinations was drawn from 6-week-old rats. In studies of the High-DPG and Low-DPG strain rats, bleeding for cholesterol determinations was done at a number of ages, and both male and female animals were used. To control for effects of age, sex, blood-drawing history, and time of day, each HighDPG strain rat was paired with a Low-DPG strain animal of similar sex, age, generation, and bleeding history and blood was taken at the same time. Determinations of cholesterol levels in the $F_{2}$ animals involved only males, 32-35 weeks of age, with a bleeding history of a single $1.0-\mathrm{ml}$ bleeding at 6 weeks of age. The $\mathrm{F}_{2}$ animals were chosen for cholesterol determinations because their 6-week DPG levels were either very low (Low-DPG group) or very high (High-DPG group) so that their genotype at the DPG-level-determining locus was quite certain. They were sampled from a large number of the $F_{2}$ families produced and therefore should represent a random sample of the genetic variability in the $F_{2}$ population at loci other than the DPGlevel-determining locus.

Levels of DPG were determined on trichloroacetic acid extracts of whole blood by the method of Keitt (1971). Plasma cholesterol levels were determined by a variant of the standard Liebermann-Burchard test for cholesterol (Kelsey, 1939). Hemoglobin levels were determined by the cyanmethemoglobin method.

\section{RESULTS}

Table I gives the mean and standard deviations of age-adjusted plasma cholesterol levels, 6-week DPG levels, and 6-week hemoglobin levels for 42 HighDPG strain rats and their 42 Low-DPG strain partners. Since cholesterol levels increase with age and these pairs varied considerably in age at bleeding for cholesterol determination, raw data were adjusted to the overall mean age of 33 weeks using the linear regression of cholesterol level on age for each strain. This age adjustment reduces variation due to age and therefore makes strain data and $F_{2}$ data more comparable. Table I indicates that High-

Table I. High-DPG and Low-DPG Strain Data

\begin{tabular}{|c|c|c|c|c|}
\hline Strain & $N$ & $\begin{array}{c}\text { Age-adjusted plasma } \\
\text { cholesterol } \\
\text { mean } \pm \text { sD }\left(\mathrm{mg}_{0} \%\right)\end{array}$ & $\begin{array}{c}\text { 6-week DPG } \\
\text { mean } \pm \text { SD } \\
(\mu \text { moles/g hemoglobin })\end{array}$ & $\begin{array}{c}\text { 6-week hemoglobin } \\
\text { mean } \pm \text { SD }(\mathrm{g} \%)\end{array}$ \\
\hline High-DPG & 42 & $80 \pm 14\}^{b}$ & $33.9 \pm 0.5\}^{b}$ & $12.3 \pm 0.2\}^{b}$ \\
\hline Low-DPG & 42 & $110 \pm 20\}$ & $21.1 \pm 0.5\}$ & $13.1 \pm 0.2\}$ \\
\hline
\end{tabular}

${ }^{a}$ See text for explanation.

${ }^{b} p<0.01$. 
Table II. $F_{2}$ Data

\begin{tabular}{|c|c|c|c|c|}
\hline Group & $N$ & $\begin{array}{l}\text { Plasma cholesterol } \\
\text { mean } \pm \mathrm{SD}(\mathrm{mg} \%)\end{array}$ & $\begin{array}{c}\text { 6-week DPG } \\
\text { mean } \pm \mathrm{SD} \\
(\mu \text { moles/g hemoglobin })\end{array}$ & $\begin{array}{c}\text { 6-week hemoglobin } \\
\text { mean } \pm \mathrm{SD}(\mathrm{g} \%)\end{array}$ \\
\hline High-DPG & 20 & $99 \pm 32\}^{a}$ & $35.4 \pm 0.9\}^{a}$ & $11.7 \pm 0.4\}^{a}$ \\
\hline Low-DPG & 17 & $128 \pm 25\}$ & $25.6 \pm 1.1\}$ & $12.4 \pm 0.5\}$ \\
\hline
\end{tabular}

${ }^{n} p<0.01$.

DPG strain animals, with high DPG levels and low hemoglobin levels relative to Low-DPG strain animals, have considerably lower plasma cholesterol levels than Low-DPG strain rats. Strain differences in mean plasma cholesterol, DPG, and hemoglobin levels are highly significant $(p<0.01)$. Although DPG levels were measured only at 6 weeks of age, a great deal of experience with these rat strains has shown us that, while DPG levels decrease with age, the DPG differences between strains are maintained throughout the rats' lifetimes. There was some indication in the raw data that there might be a sex effect on cholesterol levels, but a two-way analysis of cholesterol levels in the two sexes and the two strains indicated that the variable of sex did not contribute significantly to the total variability in cholesterol levels in these rats.

The data for the $F_{2}$ animals are given in Table II. Since all of the $F_{2}$ animals were bled for cholesterol determinations at 32-35 weeks of age, no age adjustments were necessary. Differences in mean plasma cholesterol levels in these two groups of $\mathrm{F}_{2}$ rats are highly significant $(P<0.01)$, in the same direction as the strain data. Six-week DPG and hemoglobin levels in this group of $\mathrm{F}_{2}$ males are similar to those shown in Table I for strain animals, and again $t$ tests indicate that the means for both variables are significantly $(P<0.01)$ different in the two groups.

\section{CONCLUSIONS AND DISCUSSION}

The data presented in Table I show that relatively low cholesterol levels and the allele for High-DPG levels occur together in High-DPG strain rats, while relatively high cholesterol levels occur in strain animals homozygous for the Low-DPG allele. This association could have been due to inadvertent fixation of different cholesterol-level-determining alleles in the two strains as a result of the inbreeding which necessarily occurred during the development of the strains. The fact that this association was present in $F_{2}$ animals as well as in strain rats virtually eliminates the possibility that the association is due to inadvertent fixation of cholesterol-determining alleles, unless this gene(s) is 
closely linked to the DPG-level-determining gene. This is because $F_{1}$ animals are heterozygous at all loci at which the strains differ and the cross of $F_{1}$ animals with one another leads to random reassortment of genes. The distribution of cholesterol-level-determining genes in $\mathrm{F}_{2}$ animals with HighDPG levels should not be significantly different than their distribution in $\mathrm{F}_{2}$ animals with low DPG levels unless the genes are linked (on the same chromosome). We hope to deal with the question of linkage in future studies.

Assuming that the association is not due to genetic linkage, three additional explanations for the association can be considered. The first is our working hypothesis, namely that the DPG differences cause the cholesterol differences. This could occur as a result of oxygen transport differences brought about by different DPG levels. The High-DPG animals have a lower hemoglobin oxygen affinity and thus more efficient oxygenation. Second, the DPG-cholesterol relationship could theoretically result from plasma cholesterol levels somehow altering red cell metabolism to produce different DPG levels. This seems unlikely since we have observed that high-cholesterol diets do not appear to have an effect on the DPG levels and erythrocyte oxygen affinity curves of rats and rabbits. We have administered highcholesterol diets to 24 rabbits and 36 rats, sufficient to cause a severalfold increase in serum cholesterol, and have not seen any effect on DPG levels. Third, the DPG-cholesterol relationship could arise from independent effects of an additional factor on both DPG levels and cholesterol levels. This factor could be the activity of the glycolytic control enzyme, phosphofructokinase (PFK). Studies of levels of red cell glycolytic intermediates in the strains (Noble and Brewer, 1972) and in the $F_{1}$ and $F_{2}$ animals (unpublished data) strongly suggest that the DPG-level-determining gene acts by a mechanism involving differences in the in vivo activity of red cell PFK. This PFK activity difference could also exist in liver cells of the two types of rats and, in some way, result in differences in cholesterol metabolism.

Further study is necessary to test whether or not the cholesterol differences we have found are caused by oxygen affinity differences. However, in view of the relationship between elevated cholesterol levels and arteriosclerosis in human, the finding that genetically Low-DPG (high oxygen affinity) rats have high cholesterol levels relative to genetically High-DPG (low oxygen affinity) rats is interesting and provocative.

\section{ACKNOWLEDGMENT}

We wish to thank T. . Leonard for his valuable assistance. 


\section{REFERENCES}

Benesch, R., and Benesch, R. E. (1967). The effect of organic phosphates from the human erythrocyte on the allosteric properties of hemoglobin. Biochem. Biophys. Res. Commun. 26:162.

Brewer, G. J. (1972). Clinical implications of variation in erythrocyte oxygen affinity: A. Blood storage and B. Arteriosclerosis. In Astrup, P., and Rorth, M. (eds.), Oxygen Affinity and Red Cell Acid Base Status, Academic Press, New York, pp. 629-645.

Brewer, G. J., Oelshlegel, F. J., Jr., and Eaton, J. (1972). Biochemical, physiological and genetic factors in the regulation of mammalian erythrocyte metabolism and DPG levels. In Astrup, P., and Rorth, M. (eds.), Oxygen Affinity and Red Cell Acid Base Status, Academic Press, New York, pp. 539-551.

Chanutin, A., and Curnish, R. R. (1967). Effect of organic and inorganic phosphates on the oxygen equilibrium of human erythrocytes. Arch. Biochem. 121:96.

Keitt, A. (1971) Reduced nicotinamide adenine dinucleotide linked analysis of 2,3-DPG. J. Lab. Clin. Med. $77: 470$.

Kelsey, H. (1939). Determination of cholesterol. J. Biol. Chem. 127:15.

Noble, N., and Brewer, G. J. (1972). Studies of the metabolic basis of the ATP-DPG differences in genetically selected high and low ATP-DPG rat strains. In Brewer, G. J. (ed.), Hemoglobin and Red Cell Structure and Function, Plenum, New York, pp. 155164.

Noble, N. A., and Brewer, G. J. (1977). Identification of a major locus contributing to erythrocyte 2,3.diphosphoglycerate variability in hooded (Long-Evans) rats. Genetics 85:669. 